\title{
Code 9: Difficulty Getting Access to Care/Side Effects
}

These results clearly demonstrate what major surgery patients have had, and the requirement for ongoing care, despite discharge from hospital.

- 'There is a significant cost if prostate cancer is not treated early.'-ID 3

- 'Ethnic minorities need to have ED addressed too.'-ID 1

- An immediate standardized pathway after incontinence has been treated needs to be in place.'-ID 1

This code highlights a number of issues. Care after major surgery is often difficult to get, especially over weekends (Table 54.1). As part of the pathway, preoperative assessment is needed as specified, in order to manage patient expectations regarding post-operative side effects of therapy. Additionally, better access to care needs to be arranged post operatively, which can be facilitated via a pathway. This also gives us the timing for a pathway- after incontinence issues have been resolved.

Table 54.1 Code 9: Difficulty getting access to care/side effects

\begin{tabular}{l|l}
\hline & Patient quotes using Patient Idenfitication Number \\
\hline Post-operative support & 'Weekends are a problem to get support.'-ID 2 \\
& 'We need 24/7 care.'-ID3 \\
\hline Post-operative support & 'Major surgery hits you hard after the operation'-ID5 \\
\hline
\end{tabular}

